\title{
National Perinatal Association Guidance for Palivizumab Prophylaxis Increases Coverage to that Provided by the FDA Indication
}

Mitchell Goldstein ${ }^{1}$, Benjamin Harding ${ }^{1}$, and Elba Fayard ${ }^{1}$

${ }^{1}$ Loma Linda University School of Medicine

January 24, 2021

\author{
Abstract \\ Recommendations" \\ Mitchell Goldstein, MD \\ Division of Neonatology \\ Department of Pediatrics \\ Loma Linda University Children's Hospital \\ 11175 Campus Street, Suite \#11121 \\ Loma Linda, CA 92350 \\ mgoldstein@llu.edu \\ Benjamin Harding, MD \\ Division of Neonatology \\ Department of Pediatrics \\ Loma Linda University Children's Hospital \\ 11175 Campus Street, Suite \#11121 \\ Loma Linda, CA 92350 \\ bharding@llu.edu \\ Elba Fayard, MD \\ Division of Neonatology \\ Department of Pediatrics \\ Loma Linda University Children's Hospital \\ 11175 Campus Street, Suite \#11121 \\ Loma Linda, CA 92350
}

This editorial is an invited response to "Adherence to the 2014 American Academy of Pediatrics Palivizumab Prophylaxis 
efayard@llu.edu

Disclosures: The authors have no relevant conflicts of interest.

Keywords: RSV, palivizumab, prophylaxis, NPA, AAP

Palivizumab is a synthetic monoclonal antibody targeting the F-protein spike of the Respiratory Syncytial Virus. First approved by the United States Food and Drug Administration (FDA) in 1998, its indicated usage mirrored the American Academy of Pediatrics, Committee on Infectious Disease (AAP- COID) guidance through the first few years following its approval. At that time, primarily based on expert opinion and cost considerations, the AAP-COID progressively decreased its guidance, culminating in a policy statement released in 2014 that excluded $75 \%$ of the patients initially covered by the FDA indication. ${ }^{1,2}$ The National Perinatal Association (NPA) first became concerned with the continued ratcheting down of coverage in 2009 when it released its first of three guidances on palivizumab prophylaxis. The intent was to provide an alternative to the AAP guidance and subsequent policy statement that increasingly deviated from the original FDA indication and failed to recognize myriad peer-reviewed studies that confirmed disease severity, prevalence, hospitalization, and response to prophylaxis.

The NPA guidance was first presented to Loma Linda University Children's Hospital (LLUCH) Neonatologists in 2009. At that time, usage of this guidance or the AAP guidance (at that time, less restrictive) was optional. The attending neonatologist would indicate which guidance the baby was to be discharged home on and the rationale for the decision. With the release of the AAP 2014 policy, the NPA guidance was encouraged, as reflected by a change in the LLUCH NICU resident manual. ${ }^{3,4}$ LLU neonatal physicians met with a local pharmacy to streamline RSV prophylaxis administration to those at-risk patients. In 2018, the most recent NPA guidance was officially adopted by LLUCH Neonatologists. ${ }^{2}$ A dot-phrase was built into the EHR to assure compliance. Year over year, RSV inpatient dose administration increased from 115 in 2017-2018, to 117 in 2018, to 176 in 2019-2020. Outpatient dosing increased from 0 (reported) in 2017-2018, to 175 in 2018-2019, and 487 in 2019-2020. $(\mathrm{p}<0.01)$

Although the current study provides information regarding the demographics of the patients who received palivizumab before and after changes in the AAP COID policy, compliance remains an issue to any guidance or policy. The unintended effects of a restrictive policy often extend beyond the boundaries of its intended advice. In many locations, palivizumab cannot be obtained for even those patients who clearly qualify by both metrics. ${ }^{5}$ Unrecognized morbidities are substantial. Most studies do not adequately capture cough, wheeze, secondary infection, and failure to thrive. ${ }^{6}$ Lost productivity from parents and other caregivers having to remain at home with a sick child is not considered. Hospital billing data versus actual payment and palivizumab cost data versus actual payment can be manipulated to denigrate a highly effective biologic with a demonstrated safety profile.

In sum, the authors have presented compelling data regarding palivizumab's efficacy but have just begun to scratch the surface. Loma Linda is the only level 4 NICU in San Bernardino County, the largest in the state. As they pointed out, many inpatients from LLUCH NICU do wind up seeking care at alternate centers and may receive doses of palivizumab from other pharmacies. Extended to other centers, differences in practice patterns and palivizumab administration may reveal gaps in coverage. Changes in the practice pattern of the LLUCH Neonatologists and administration of palivizumab in subsequent years may provide an even more compelling argument for prophylaxis according to FDA indication.

References:

1. Goldstein M, Krilov LR, Fergie J, et al. Respiratory Syncytial Virus Hospitalizations among U.S. Preterm Infants Compared with Term Infants Before and After the 2014 American Academy of Pediatrics Guidance on Immunoprophylaxis: 2012-2016. Am J Perinatol.2018;35(14):1433-1442.

2. Goldstein M, Phillips R, DeVincenzo J, et al. National Perinatal Association 2018 Respiratory Syncytial Virus (RSV) prevntion clinical practice guideline: An evidence-based interdisciplinary collaboration. Neonatology Today. 2017;12(10):10. 
3. American Academy of Pediatrics Committee on Infectious D, American Academy of Pediatrics Bronchiolitis Guidelines C. Updated guidance for palivizumab prophylaxis among infants and young children at increased risk of hospitalization for respiratory syncytial virus infection.Pediatrics. 2014;134(2):415-420.

4. Goldstein M, Merritt T, Phillips R, Martin G, Hall S, Yogev R. National Perinatal Association 2015 respiratory syncytial virus (RSV) prevention guideline. Neonatology Today. 2014;9(11):1-11.

5. Goldstein M, Krilov LR, Fergie J, et al. Unintended Consequences Following the 2014 American Academy of Pediatrics Policy Change for Palivizumab Prophylaxis among Infants Born at Less than 29 Weeks' Gestation. Am J Perinatol. 2020.

6. Blanken MO, Rovers MM, Molenaar JM, et al. Respiratory syncytial virus and recurrent wheeze in healthy preterm infants. The New England journal of medicine. 2013;368(19):1791-1799. 